\title{
Recherches concernant l'écoulement permanent alimentant un puits gravitaire*
}

\author{
PAR HOWARD P. HALL \\ English text, p. 8
}

Se reporter au texte anglais p. 8, pour les illustrations et la bibliographie

\begin{abstract}
Etude au moyen d'essai sur modèle réduit à grande échelle de l'écoulement au voisinage immédiat d'un puits gravitaire atteignant la couche imperméable et présentation des résultats obtenus. Examen particulier de l'allure de l'écoulement dans la région où la pente de la surface libre devient si prononcée que les hypothèses de Dupuit ne sont plus valables.

Conception et réalisation du modèle, description des essais et des mesures, résultats obtenus. La méthode de relaxation: modifications apportées par $\mathrm{Y}_{\mathrm{ANG}} \dot{a}$ la méthode de SoUTHweLL; application à différents cas essayés, comparaison des résultats obtenus abec les résultats expérimentaux. Correspondance satisfaisante dans la mesure où l'on tient compte des effets capillaires.
\end{abstract}

\begin{abstract}
Importance des effets capillaires au voisinage de la paroi du puits.

Présentation d'un ensemble de formules empiriques permettant de tracer la surface libre dans un plan radial d'un puits donné.

Conclusions : validité de la méthode de relaxation modifiée par YANG pour étudier l'alimentation d'un puits gravitaire; difficulté de la solution qui exige un contrôle soigneux des conditions allx limites près de la surface libre; déformation de l'écoulement au voisinage de son intersection avec la paroi du puits (par suite des effets capillaires); possibilité de tenir compte de ces effets capillaires dans la méthode de relaxation en choisissant judicieusement les conditions aux limites.
\end{abstract}

\section{I. - INTRODUCTION}

\section{A) Définitions}

L'alimentation d'un puits par une nappe peut schématiquement être assimilée à l'écoulement à trois dimensions, autour d'un axe de symétrie, d'un fluide homogène en milieu poreux.

Si la couche inférieure sur laquelle s'écoule la nappe n'est pas surmontée d'une couche imperméable, l'eau de la nappe est en contact avec l'air, et il existe dans l'écoulement une surface imaginaire en tous les points de laquelle la pression correspond à la pression atmosphérique. Si l'on néglige les phénomènes capillaires, cette surface imaginaire constitue la limite supérieure de l'écoulement, c'est-à-dire le «niveau de la nappe», ou «la surface libre ». Dans de telles conditions, le mouvement des particules liquides dépend de la pesanteur aussi bien que de la charge hydraulique; on a à faire à ce que l'on appelle «un écoulement à surface libre ". Un puits gravitaire est un puits alimenté dans de telles conditions.

$\left(^{\star}\right)$ D'après une thèse soumise par l'auteur, en décembre 1950, à la « Faculty of Arts and Sciences of Harvard University ».
On verra que même après l'établissement d'un régime permanent, l'intersection de la surface libre et de la paroi d'un puits gravitaire est située audessus du niveau de l'eau à l'intérieur du puits. La surface cylindrique découpée sur la paroi du puits par ces deux niveaux est appelée : surface de suintement.

\section{B) Les buts de l'étude}

Très récemment encore, les méthodes de calcul les plus exactes, pour l'écoulement permanent autour d'un puits gravitaire, voyaient leur validité limitée aux zones dans lescuelles la pente de la surface libre était assez faible (moins de $15 \%$ environ) pour justifier l'adoption, à la base de calcul,

(*) N.d.T. - L'expression « puits gravitaire 》 n'est pas employée couramment; mais elle nous parait constituer un néologisme d'autant plus intéressant qu'elle se rapproche beaucoup de l'expression consacrée anglaise : «gravity well 》.

En général, en français, on parle plutôt de «puits ordinaire» ou de «puits à surface libre». 
des hypothèses fondamentales de Dupuit [2] $\left(^{*}\right)$. Grâce à la mise au point de la méthode de relaxation de Southwell $[4,5]$, et à l'adaptation qui en a été faite au cas qui nous intéresse, en particulier dans les études de S. T. Yang [7], il semble possible maintenant de se livrer à un calcul théorique précis de ce type d'écoulement.

Les travaux qui sont présentés dans cet article avaient pour objet de chercher à préciser les caractéristiques de l'écoulement permanent au voisinage d'un puits gravitaire en s'appuyant sur des essais sur modèle réduit à échelle relativement grande; on se proposait ensuite d'établir une correspondance satisfaisante entre les résultats obtenus à la suite des essais, d'une part, et ceux fournis par le calcul théorique, d'autre part. Une telle correspondance avait le double avantage :

- de prouver que certaines des difficultés les plus gênantes des premiers modèles à sable peuvent être surmontées;

- de mettre en évidence la possibilité d'exploiter la méthode de relaxation sans se heurter à des calculs inextricables et interminables.

\section{II. - LE MODÈLE}

\section{A) La conception du modèle}

1. Dispositions gÉnérales. - Le modèle devait représenter, sur un secteur de $15^{\circ}$, la zone d'alimentation d'un puits gravitaire occupant toute la hauteur de la nappe. Le schéma de la figure 1 donne les dimensions principales. Les parois latérales, PTWS et QUVR, représentent des sections verticales radiales passant par l'axe, MN, du puits; la section PQRS, face aval du modèle, représente une partie de la paroi du puits; la section TUVW, face amont du modèle, représente une fraction de surface verticale cylindrique entourant le puits à une certaine distance de celui-ci; la surface SRVW représente un substratum horizontal imperméable. Ainsi la distance $\mathrm{MT} \quad(=\mathrm{MU}=\mathrm{NW}=\mathrm{NV})$ correspond au rayon $\mathrm{R}$ de la zone au-delà de laquelle la pente de la ligne d'eau est nulle, ou négligeable. La distance $\mathrm{MP}(=\mathrm{MQ}=\mathrm{NS}=\mathrm{NR})$ correspond au rayon $\mathrm{du}$ puits $r_{0}$.

2. Les DIMENStons du Modèle. - Il était souhaitable de choisir de grandes dimensions afin de faciliter les observations dans le voisinage du puits et de réduire l'influence des phénomènes capillaires. Il était également souhaitable de prendre pour 0 une petite valeur (fig. 1) afin que soit justifiéc l'adoption de surfaces planes pour représenter plus simplement les surfaces cylindriques constituant les sections amont et aval du modèle. D'autre part, la section aval devait avoir une largeur suffisante (PQ ou SR de la fig. 1) pour que les tolérances d'usinage normales n'introduisent pas d'irrégularités trop sensibles; enfin le rapport $R / r_{0}$ devait être suffisamment élevé pour que la section verticale d'entrée constitue une approximation valable de la surface équipotentielle située à une distance correspondante d'un puits de ce type.

La valeur $\theta$ fut fixée à $15^{\circ}$, et le rapport $\mathrm{R} / r_{0}$ à $16\left(^{* *}\right)$. Le rayon du puits était $r_{0}=4,8$ pouces $(\# 12,2 \mathrm{~cm})$, et la distance entre l'axe du puits et

$\left.{ }^{\star}{ }^{\star}\right)$ Les chiffres entre [ ] renvoient à la bibliographie en fin du texte anglais.

$\left({ }^{\star}\right)$ Suivant les résultats obtenus par BaBirt et $\mathrm{C}_{\text {ALD }}$ wELL [1], les surfaces équipotentielles sont des cylindres à peu près verticaux à une distance comprise entre 15 et 20 fois le rayon du puits. la section amont était $\mathrm{R}=76,8$ pouces (\#195 $\mathrm{cm}$ ). Dans ces conditions la largeur de la section aval s'élevait à 1,26 pouce $(32 \mathrm{~mm})$, et celle de la section amont à 20,05 pouces $(512 \mathrm{~mm})$.

3. Mmleux poreux. - C'est du sable type d'Ottawa $\left(^{*}\right)$ qui fut choisi comme conciliant au mieux le souci d'économic et l'obligation de disposer des propriétés physiques voulues. La très grande régularité de la granulométrie et la forme presque sphérique des grains réduisaient l'importance des erreurs pouvant provenir d'un manque d'homogénéité et d'une stratification.

4. Gradient hydraulique et débit. - La formule de Dupurt-Threm permit d'estimer la valeur moyenne du gradient hydraulique au niveau de la section aval. En supposant à cet effet que $h_{0}=0$ et que $\mathrm{Z}_{0}=(0,5) \times(\mathrm{H})=2 \mathrm{ft}(\# 60 \mathrm{~cm})$, on fixe à environ 3,5 la valeur moyenne du gradient sur la section aval. Des essais effectués sur du sable d'Ottawa afin de préciser le domaine de validité de la loi de DARcy montrèrent que la relation entre Ia vitesse et le gradient hydraulique cessait d'être linéaire pour une valeur du gradient voisine de 3 . Le sable essayé avait le même module des vides que celui disposé dans le modèle. En appliquant la formule de Dupurt-THiem avec $k=0,4 \mathrm{~cm} / \mathrm{s}$, le débit maximum à prévoir fut fixé à $250 \mathrm{~cm}^{3} / \mathrm{s}$.

5. Détails. - La figure 2 représente le modèle en perspective. Afin de la rendre plus claire, un seul piézomètre a été représenté, et on a éliminé tous les détails concernant les dispositifs de circulation d'eau.

Les parois latérales étaient transparentes afin de faciliter les conditions régnant au niveau de la surface libre; la «lucite» (plexiglass) fut choisie de préférence au verre en raison de ses facilités de manipulation et d'usinage.

Afin d'éviter que les parois en lucite prennent sous la charge une flèche excessive, elles s'appuyent sur quelques armatures en acier prévues à cet effet. Ces armatures étaient fixées à la plaque de base du modèle d'une part, d'autre part elles étaient liées l'une à l'autre par-dessus le modèle.

Le châssis-support fut constitué d'une tôle d'acier de $1 / 4$ de pouce d'épaisseur $(6,35 \mathrm{~mm})$,

(U.) Ottawa est ici une petite ville de l'Etat d'Iminois 
fixée à deux gros fers à larges ailes, ce qui fut jugé suffisant pour éviter des déformations excessives.

$\mathrm{Au}$ niveau des sections amont et aval les parois furent réalisées en matière plastique plutôt qu'en métal, afin d'atténuer les risques d'électrolyse ou de rouille.

A l'extrémité aval il était souhaitable de disposer un gros tube vertical afin d'áugmenter la rigidité de la charpente du modèle en un point où les déformations risquaient d'être les plus gênantes. A l'extrémité amont il était suffisant de disposer une gouttière verticale en tôle mince à peu près semicylindrique. Il convenait dans ce cas d'adopter une section plus grande afin de permettre d'alimenter le modèle avec le débit maximum nécessaire tout en évitant l'apparition d'une turbulence au niveau de la section d'entrée.

Il convenait également d'adopter pour l'alimentation du modèle un système en circuit fermé afin de limiter le volume d'air susceptible de s'introduire pendant le déroulement des essais. Ce système comportait une conduite de retour joignant la conduite verticale aval à une pompe centrifuge, puis à un débitmètre après lequel l'eau était réintroduite dans la cuve d'alimentation par l'intermé- diaire d'un tube poreux destiné à atténuer les effets de la turbulence.

Les joints et les étanchéités se présentaient comme devant être la source du plus grand nombre d'ennuis: les joints en caoutchouc furent choisis comme constituant la plus satisfaisante des étanchéités.

\section{B) Réalisation du modèle - Préparation des essais.}

Les figures 4 à 9 donnent des détails de réalisation du modèle. Le modèle était d'abord rempli d'eau chaude; et ceci pour la raison suivante :

L'eau du modèle était maintenue relativemen1 chaude jusqu'à ce que les essais soient sur le point de commencer; de cette façon, une fois commencée la phase de refroidissement, l'eau se trouvait non saturée en air dissous lorsqu'elle atteignait la température extérieure. Ainsi, au fur et à mesure du déroulement des essais, l'eau avait tendance à absorber de l'air, plutôt qu'à en dégager en risquant de créer des poches d'air.

Le modèle une fois rempli d'eau, le sable était mouillé à saturation par très faibles quantités et mis en place sous l'eau.

\section{III. - LES ESSAIS}

\section{A) Modalités d'exploitation}

1. BUT DES ESSAIS. - Le but principal des essais sur modèle était la détermination du réseau correspondant à l'écoulement permanent dans le cas dc l'alimentation d'un puits gravitaire - ceci à l'intérieur de la zone représentée sur le modèle. On se proposait par ailleurs :

- D'étudier la validité de la loi de DARcy pour le calcul du débit;

- De déterminer l'influence d'une frange capillaire sur le débit, d'une part, sur la forme du réseau, d'autre part;

- D'étudier quelles répercussions pourraient avoir le degré et l'uniformité de la saturation du sable.

2. Coefficients caractéristidques. - Le réseau dépend de 4 variables indépendants : $\mathrm{R}, r_{0}, \mathrm{H}$, et $h_{0}$ (fig. 1). Ainsi, un problème donné peut être défini par les valeurs de 3 coefficients indépendants quelconques, appelés coefficients caractéristiques, et contenant ces variables.

Dans le cas qui nous occupe, les variables avaient été groupées suivant les rapports $\mathrm{R} / r_{0}$, $\mathrm{H} / r_{0}$ et $h_{0} / \mathrm{H}$. La valeur du premier de ces rapports fut uniformément fixée à 16 pour tous les essais. La valeur du second, $\mathrm{H} / r_{0}$, était maintenue à une valeur constante voisine de 10 , en fixant le niveau dans le tuyau vertical d'entrée. On donnait au troisième, $h_{0} / \mathrm{H}$, pour les différents essais, des valeurs différentes prises dans une série de valeurs; on réglait convenablement à cet effet le niveau d'eau dans le tube vertical de restitution. Toute la gamme des valeurs possibles, entre 0 et 1 , a été explorée au cours des essais.

3. Programme Des essiss. - Il y a en quatre séries d'essais. La série A comporta 8 essais, le sable ayant été préalablement mis en place, comme l'in. dique le paragraphe II B. 2. $\mathrm{H}$ étant maintenu à 48,0 pouces $(122 \mathrm{~cm}), h_{0}$ fut réduit de 42,0 pouces $(107 \mathrm{~cm})$ au premier essai jusqu'à zéro au dernier, par fractions successives de chacune 6 pouces $(15,2 \mathrm{~cm})$.

Après achèvement de la série $A$, le modèle fut vidé, puis rempli à nouveau d'eau sans en retirer le sable. La série $B$ comporta trois essais réalisés au cours du remplissage. $H$ étant toujours maintenu à la valeur de 48,0 pouces $(122 \mathrm{~cm})$, on donna à $h_{0}$ les valeurs successives $0,12,0$ et 24,0 pouces $(30,2$ et $60,4 \mathrm{~cm}$ ).

La série $C$ reprit les mêmes essais de la série $B$, mais dans l'ordre inverse, après remplissage complet du modèle.

La série $D$ fut une répétition de la série $C$, la première eau ayant été remplacée par une eau dont on avait réduit la tension superficielle par un traitement chimique convenable.

\section{B) Mesures}

1. Mise an table. - Le tableau I résume les mesures importantes faites au cours des essais, à l'exception des lectures au piézomètre. On y a adjoint une colonne donnant les valeurs de la hauteur 
$z_{0}$ du point d'intersection de la surface libre et de la surface du puits, bien que ces valeurs résultent du diagramme des lectures piézométriques plutôt que de l'observation directe.

2. Lignes de courant. - Les figures 10 et 11 sont des vues photographiques des lignes de courant colorées, prises pendant l'essai 8 de la série A. Quelques-unes de ces lignes sont également représentées sur la figure $15\left(^{*}\right)$. Les lignes de courant colorées, observées pendant l'essai A 6, sont données sur la figure 14. Les figures $10,11,14$ et 15 montrent distinctement une zone d'inflexion sur ces lignes, qui sont toutes légèrement concaves vers le haut, au voisinage de la section aval. A noter que la ligne de courant supérieure de l'essai A-8 (fig. 15), qui passe bien en dedans de la frange capillaire, présente un coude brusque près de la section aval et passe sous la surface libre.

Le tableau II contient les valeurs mesurées qui ont servi à la détermination des vitesses relatives des lignes de courant. Le plus souvent les mesures de temps sont exactes à environ un dixième de minute près; mais le degré d'exactitude diminue vers l'extrémité aval où les bandes colorées sont plus diffuses et deviennent par conséquent plus difficiles à détecter alors que la vitesse augmente.

3. Lectures au piézomètre. - Elles formaient la majeure partie des mesures. Sur les figures 12 à 15 toutes les valeurs sont marquées à l'endroit de la paroi en lucite où elles ont été relevées. Les surfaces libres ont été tracées d'après les lectures piézométriques; la limite supérieure de la frange capillaire a été déduite des observations directes.

\section{C) Résultats des essais}

1. GÉnÉralités. - Le degré de saturation le plus élevé correspond à la série $\mathrm{A}$. Ce fut le seul cas où l'eau fut introduite dans le modèle avant le sable, qui était disposé sous l'eau après avoir été saturé, par petites quantités, dans le vide. Ni le nombre des poches d'air, ni leur répartition n'ont introduit de grosses erreurs jusqu'au dernier essai de la série A.

Dans le cas de la série $B$, puis, progressivement de moins en moins au cours des séries $\mathrm{C}$ et $\mathrm{D}$, la difficulté majeure provenait de la distribution non uniforme des poches d'air. Cette difficulté a été, pour une large part, surmontée dans les séries $C$ et $D$; mais les chiffres relevés dans la série $B$ indiquent le peu de valeur des résultats, qui prouvent uniquement que même avec un grand modèle et dans un milieu poreux d'un grain aussi gros que le sabletype d'Ottawa, on ne saurait obtenir une perméabilité réelle sensiblement uniforme en introduisant directement l'eau dans la masse de sable, sans autres précautions.

Les essais de la série A se sont déroulés dans des conditions à peu près idéales. On a donc pris dans cette série les résultats des essais 2,4 et 5 comme base de comparaison avec les résultats de l'analyse

(*) Le quadrillage des figures $12,16,22,25$ et 27 reprisente le réseau à mailles carrées de 2 pouces $(50,8 \mathrm{~mm})$ qui était tracé sur les parois latérales du modèle. théorique; et c'est sur les résultats de l'ensemble de la série A que s'appuient toutes les considérations qui suivent. La comparaison des résultats des séries $B, C$ et $D$ à ceux donnés par la série $A$, montre l'importance relative des facteurs tels que le degré de saturation, l'action capillaire, l'uniformité réelle du sable.

2. Réseaux D'Écoulement. - Les figures 12 à 14 montrent les réseaux correspondant aux essais 2, 4 et 6 de la série $A$, tels qu'ils ont été déduits de lobservation. Une famille de courbes équipotentielles, partageant la zone de l'écoulement en dix bandes présentant la même chute de potentiel, a été tracée, après interpolation des lectures piézométriques. Dans le $3^{\circ}$ essai, ces intervalles correspondent à des hauteurs d'eau respectives de 1,2, 2,4 et 3,6 pouces $(3,6,1$ et $9,2 \mathrm{~cm})$. Dans chaque cas on déterminait la surface libre en recherchant les points d'intersection des lignes équipotentielles avec les niveaux correspondants des piézomètres, puis en faisant passer une courbe par cette série de points. Les lignes de courant de la figure 14 ont été tracées d'après le chemin suivi par des traînées de colorant bleu injecté en ces points.

La figure 15 renseigne sur l'essai 8 , de la série $A$, comme les figures 12 à 14 renseignaient sur les essais 2 , 4 et 6 . En passant de l'essai 7 à l'essai 8 , les lectures au piézomètre sont demeurées invariables pendant plus d'une journée. Après deux jours, une seconde série de lectures piézométriques pour l'essai 8 accusait une augmentation générale. Les augmentations les plus fortes étaient notées à l'angle inférieur de l'extrémité aval : elles devaient manifestement être attribuées à la formation de poches d'air dans cette région. Ce phénomène était tout à fait local et ne fut observé nulle part ailleurs. Il n'en résultait aucune modification appréciable de la position de la surface libre, ce qui prouve que la perturbation n'était pas suffisante pour entraîner une déformation grave de l'ensemble de l'écoulement.

Le fait que les piézomètres n'ont pas enregistré de changement immédiat appréciable au moment où l'essai 7 s'achevait et où commençait l'essai 8 a été le premier indice d'un dégagement d'air appréciable. Si des conditions idéales avaient été réalisées, on aurait du assister à une réduction générale des lectures des piézomètres, réduction allant en s'accentuant depuis des valeurs négligeables, près de la section d'entrée, jusqu'à plusieurs pouces au point d'intersection de la plaque de base et de la section de sortie. Toutefois comme les poches d'air commençaient déjà à créer une forte résistance à l'écoulement, surtout au point où l'écart à prévoir était le plus grand, on peut conclure qu'à la baisse générale des hauteurs piézométriques s'opposait un effet d'obstruction causé par ces poches d'air.

Les figures 10 et 11 apportent d'autres indices de l'influence des variations locales de la perméabilité réelle. La vue de la figure 10 a été prise plusieurs heures après le début de l'essai 8 . La figure 11 qui comprend trois nouvelles lignes de courant, a été obtenue un jour plus tard, après injection de colorant bleu par les huits points de départ, à l'exception du point supérieur. En comparant la figure 10 à la figure 11, on voit d'abord que les trois dernières 
lignes ne concordent pas avec les cinq premières; on constate ensuite que ces dernières ont ellesmèmes dévié, plus ou moins, depuis la veille.

On a constaté que les lignes de courant, qui avaient été attirées dans la frange capillaire, finissent par retourner à la région située sous la surface libre avant d'atteindre la section de sortie. Les deux lignes supérieures de la figure 10 ont été reproduites sur la figure 15 pour rendre ce fait visible.

Notons que la surface libre ne forme pas ligne de courant quand il existe une couche capillaire.

Ainsi, les lignes équipotentielles des figures 12 à 15 ne coupent pas nécessairement la surface libre sous un angle droit. L'écart, d'ordinaire faible, devient très apparent aux environs de la section de sortie.

3. Vitesses. - La précision des mesures reproduites au tableau II suffit tout au plus à donner des renseignements qualitatifs sur les vitesses. Ainsi, le temps que mettait le colorant bleu pour atteindre la section de sortie, en partant du point d'injection était minimum pour la ligne de courant la plus proche de la base et maximum pour les lignes les plus éloignées.

En comparant la vitesse à lacuelle le colorant avançait dans la frange capillaire avec celle de sa progression à travers la région en dessous de la surface libre, on arrivait à estimer la part qui revenait à la frange capillaire dans le débit total.

4. L'ÉcoUt_ement DANS LA FRANGe CAPILlaire. En considérant le tableau II, on note entre les deux lignes de courant supérieures un écart anormal des durées de parcours depuis les points d'injection jusqu'à la section de sortie. Celle de ces deux lignes qui part du point 1 (voir tableat II) est bien à l'intérieur de la frange capillaire, tandis que l'autre, partant du point 2, est très voisine de la surface libre. Comme le point 2 se trouvait à 2 pouces $(5,1 \mathrm{~cm})$ en dessous du niveau dans le tube vertical d'alimentation, on admet que le volume d'eau passant dans la frange capillaire équivalait à celui qui traversait la partie supérieure, haute de deux pouces $(5,1 \mathrm{~cm})$, de la section d'entrée. Si l'on admet que les vitesses étaient uniformes à l'entrée, on voit que le débit à travers la frange capillaire représente environ $4 \%$ du débit total. De plus, la concordance des variations du débit total, tout au long de la série $A$, avec les indications de l'équation de Dupurt-THrem, indiquait que les effets de la frange capillaire restaient à peu près les mêmes, quelle que soit la perte de charge.

On en déduisit que l'accroissement du débit, dû à la présence d'une frange capillaire, était sensiblement équivalent au rapport entre la demi-épaisseur de cette couche ct la charge totale à l'entrée; on avait autrement dit :

où :

$$
\frac{(\Delta Q)_{c}}{Q}=\frac{h_{c}}{2 \mathrm{H}}
$$

$(\Delta Q)_{c}=$ est l'accroissement de débit dû à la présence de la couche capillaire;

$$
h_{c}=\text { l'épaisseur moyenne de la couche capil- }
$$

\section{$\mathrm{H}=$ la profondeur de l'eau à l'entrée; \\ $Q=$ le débit tel qu'il résulte de l'équation de Dupuit-Thiem.}

5. Coefficient de perméabilité. - D'autres études $[6,1]$, avaient montré que le coefficient de perméabilité effectif réel moyen peut être déterminé avec assez de précision à partir de l'équation de Dupurt-Threm. On peut écrire celle-ci :

$$
k_{\mathrm{T}}=\frac{\mathrm{Q} \log _{e}\left(\mathrm{R} / r_{0}\right)}{\pi \mathrm{H}^{2}\left(1-\lambda^{2}\right)}
$$

où $k_{\mathrm{T}}$ est le coefficient effectif moyen de perméabilité à $\mathrm{T}^{\circ} \mathrm{C}$, et $\lambda=h_{0} / \mathrm{H}$.

Toutefois, le modèle ne représente que la $24^{\mathrm{c}}$ partie environ de la zone entourant le puits; les débits observés devraient être corrigés de l'effet de la frange capillaire et le coefficient de perméabilité ramené à la valeur correspondant à $20^{\circ} \mathrm{C}$. L'équation (2) devient ainsi :

$$
k_{20}=\frac{24 \mathrm{Q}_{\mathrm{T}}\left(1-h_{c} / 2 \mathrm{H}\right)}{\pi \mathrm{H}^{2}\left(1-\lambda^{2}\right)}\left[\log _{c}\left(\frac{\mathrm{R}}{r_{0}}\right)\right]\left(\frac{\mu_{\mathrm{r}}}{\mu_{20}}\right)
$$

où $Q_{\text {T }}$ est la valeur $d u$ débit sur le modèle à la température de $\mathrm{T}^{\circ} \mathrm{C}$.

et $\mu_{T}, \mu_{20}$ les coefficients de viscosité de l'eau à 'To et à $20^{\circ} \mathrm{C}$.

Le tableau III contient les valeurs obtenues par la résolution de l'équation (3) pour les sept premiers essais de la série «A ». La valeur moyenne de $\boldsymbol{k}_{20}$ qui en résulte est $11 \%$ plus faible qu'une autre valeur tirée d'une série d'essais directs dans des conditions similaires. Il s'ensuit que le degré de saturation obtenu avec le modèle de la série A était inférieur à celui qui résultait des essais de perméabilité effectués directement, sur une échelle bien plus petite.

La bonne concordance entre les valcurs du tableau III montre que les dégagements d'air n'avaient pas grande influence sur l'homogénéité réelle du sable pendant les sept premiers essais de la série A.

De la rapidité avec laquelle les dégagements d'air introduisaient des erreurs au cours de l'essai, on peut déduire que le manque d'uniformité de l'écoulement dépendait beaucoup plus de l'entraînement éventuel de l'air dans la conduite de retour que de la durée pendant laquelle l'eau était exposée à l'action de l'air. D'après les valeurs contenues au tableau III on voit que le contact permanent de l'eau avec l'air, à l'intérieur des deux tubes verticaux et à l'intérieur du sable, n'a pas créé de troubles sensibles pendant les quatre semaines qu'ont duré les essais; mais quand le niveau de l'eau a été réduit à zéro dans le tube vertical aval, par conséquent quand la conduite de retour (fig. 3) a cessé de débiter à plein, les poches d'air se sont développées considérablement en deux jours.

La grande diversité des conditions accompagnant les essais des séries A, B, C et D apparaît dans les valeurs que prend le coefficient effectif de perméabilité moyen, porté sur le tableau III. Dans la série $B$, pendant laquelle on n'a pas cherché à 
réduire les dégagements d'air, la valeur de ce coefficient moyen est tombée de $22 \%$ en moyenne en dessous de la valeur notée pendant la série A. Pour les séries $C$ et $D$, on avait veillé à réduire les dégagements d'air autant qu'il était possible sans recourir à des décharges et à des recharges successives du sable du modèle. On obtenait ainsi des degrés de saturation jugés équivalents pour les deux séries et on pouvait s'attendre à des conditions de perméabilité comparables. Les valeurs du coefficient de perméabilité observées ainsi ne diffèrent respectivement que de 1 et $3 \%$ de celle de la série A.

On peut donc en déduire que le remplacement régulier de l'eau dans le modèle par de l'eau plus chaude introduite graduellement pendant une longue période de temps, sous un écoulement constant, convient effectivement lorsqu'il s'agit de limiter suffisamment les dégagements d'air pour prévenir de fortes variations du coefficient de perméabilité réel.

6. LES SURFACES LIBRES. - L'examen des surfaces libres dans les huit essais de la série A, montre l'influence décroissante des abaissements successifs de 6 pouces $(5,2 \mathrm{~cm})$ du niveau aval.

Les valeurs portées sur le tableau $I$ pour la série $B$ montrent que les surfaces libres atteignaient, pour cette série, un niveau supérieur à celui de la série A; l'influence de la frange capillaire était pourtant moindre puisque sa profondeur représentait un élément actif plutôt que passif. La divergence peut être expliquée par la non-uniformité des conditions d'écoulement. Ies lectures au piézomètre et le débit variaient au cours des essais. On pouvait observer constamment, sur toute la longueur des parois latérales, un accroissement des dégagements d'air en direction de la surface libre. On se trouvait ramené au cas d'un milieu poreux non homogène, mais comme il n'était pas possible de mesurer exactement l'étendue et la nature de ce manque d'homogénéité, on n'a pas cherché à éplucher les valeurs fournies par la série $B$.

Les surfaces libres appartenant à la sèrie $\mathrm{D}$ ont été moins atteintes par cette action capillaire. Or, non seulement l'eau avait subi un traitement chimique réduisant sa tension superficielle à environ $40 \%$ de sa valeur ordinaire, mais, en plus, le degré de saturation au sein de la frange capillaire était indiscutablement moindre que dans la majeure partie de la masse de sable.

7. Comparaison des résultats obtenus avec L'ÉQUATron DE DUPUT'THIEM. - La figure 16 fournit une comparaison entre trois courbes différentes pour chacun des trois essais du groupe A. Les trois courbes en traits mixtes ont été construiLes d'après l'équation de Dupuit-Triem pour les essais 2,4 et 6 ; les trois courbes en traits interrompus représentent la hauteur piézométrique lue sur le fond du modèle; les courbes en trait plein sont les surfaces libres déduites des observations. On voit que la courbe DupuiT-Trurm exprime assez exactement la variation de la hauteur piézométrique le long de la base imperméable. L'écart le plus fort se présente pour l'essai 6 et atteint $12 \%$.

\section{IV. -COMPARAISON DES RÉSULTATS EXPERIMENTAUX ET DES RÉSULTATS THÉRIQUES DONNES PAR LA MÉTHODE DE RELAXATION}

\section{A) La méthode de relaxation}

1. GÉNÉRALITÉs. - La méthode de relaxation est une méthode d'approximations successives. Elle a été imaginée par R. V. Southwell en Angleterre, puis fit l'objet de plusieurs développements de la part de son auteur et d'un groupe de ses collaborateurs, qui l'appliquèrent à différents problèmes techniques et physiques. Pour une étude détaillée de cette méthode, on consultera les ouvrages de SOUTHWELL [4-5].

Dans l'application de la méthode à la résolution du type de problèmes considéré ici, on a donné la préférence à une de ses variantes, laquelle sera décrite succinctement dans ce qui suit.

2. Applicatron de la méthode a l'Alimentation D'UN pUITS gRAVITAIRE. - S. T. YANG [7] a appliqué la méthode de relaxation au cas particulier d'un écoulement à trois dimensions, à symétric axiale, dont un exemple-type est le problème de l'alimentation d'un puits gravitaire situé dans une couche perméable et s'enfonçant jusqu'au soubassement imperméable. C'est cette variante de la méthode de Southwelr qui a été appliquée tout an long de la partie théorique de notre étude. Nous allons indiquer ci-après brièvement les différences essentielles entre les méthodes de Yang et de SOUTHWELL.

1. YANG adopte la hauteur piézométrique plutôt que la hauteur manométrique (contrairement à Southwels.) comme fonction potentielle dont Ies valeurs aux différents sommets du réseau constituent l'objet immédiat de la recherche par la méthode de relaxation.

2. Dans les problèmes où les conditions aux limites sont inconnues au départ (comme par exemple la surface libre dans le cas présent), Southwell fait exactement coïncider la grille de relaxation avec un tracé estimé de la limite inconnue, en se servant d' "étoiles irrégulières $\gg\left({ }^{*}\right)$ aux nouds proches de cette limite; YANG, Iui, se livre à une estimation approchée de la surface libre à l'aide d'une grille dont les mailles et les brins sont intacts. Ainsi, pour un réseau à mailles carrées

(*) On entend par «étoile irrégulière» un groupe de brins, n'ayant pas tous la même longucur, et liés à un nœud voisin d'une limite irrégulière. Les brins de longucur anormale sont ceux qui relient le noud a la limite. 
(fig. 17 à 19) la limite supérieure est une ligne brisée formée d'une succession irrégulière de côtés de maille, verticaux et horizontaux, qui suivent le tracé de la surface libre. Les parties horizontales de la limite supérieure du réseau sont représentées en traits interrompus pour indiquer qu'ils sont considérés comme des demi-brins. Un demi-brin est celui qui se substitue à la moitié de la tranche correspondant au découpage normal de la surface potentielle, lorsque la tranche remplacée est située d'un côté seulement du demi-brin. Des demi-brins sont donc correctement disposés le long de la plaque de base, où on les retrouve dans toutes les solutions obtenues par la relaxation (fig. 17 à 19), par contre, à la partie supérieure du réseau on doit les considerer comme une simple approximation de la surface équipotentielle remplacée.

Ayant apporté les modifications ci-dessus à la facon de poser son problème de puits gravitaire, Yang applique la technique de relaxation imaginée par SOUTHWEL sans y toucher davantage.

3. Drscussion. - Là modification de YANG à la méthode de relaxation a été utilisée à la résolution des problèmes qui constituent l'objet de nos recherches.

A titre d'exercice préparatoire, nous avons entrepris des calculs préliminaires distincts correspondant au cas de l'essai 8 dans la série A. Dans un cas notre première estimation plaçait la surface libre trop bas, dans un autre cas, elle la situait trop haut. La mise en ouvre de la méthode de relaxation a montré que les deux solutions différaient. En effet, la surface libre correspondant à l'évaluation initiale la plus forte atteignait la section de sortie à un niveau de $10 \%$ plus élevé au-dessus de la base imperméable que suivant l'autre évaluation. Ayant tracé les lignes de courant et les équipotentielles d'après les valeurs nodales finales, nous avons constaté que les intersections n'étaient pas à angle droit dans le cas de l'estimation initiale la plus faible. Comme les intersections obtenues dans l'autre cas satisfaisaient bien à la condition d'orthogonalité, c'est la solution correspondant à l'estimation la plus forte qui a été jugée plus exacte.

Ce qui est significatif c'est que la méthode soigneusement appliquée en partant de deux valeurs initiales différentes, n'a pas conduit à des solutions identiques, bien que dans les deux cas la convergence ait été rapide dès le début. La difficulté résiderait donc dans le mode d'approximation consistant à utiliser des demi-brins tout le long de la limite, initialement inconnue, du réseau. Du moment que la surface libre n'est pas horizontale, les demibrins horizontaux introduisent une erreur de plus en plus grave à mesure que la pente de la ligne qu'ils reproduisent s'accentue; autrement dit, l'erreur augmente dans le sens où il faudrait que la précision aille en croissant, non en diminuant.

4. Conclusions. - L'emploi de la modification de Yang dans l'application de la méthode Sov'rH-
WELL élimine dans une certaine mesure le caractère automatique des corrections successives; il s'ensuit qu'en vérifiant l'exactitude d'une solution il faut non seulement veiller à ce que les forces résiduelles se réduisent à des quantités négligeables, mais aussi revoir les conditions aux limites. Il est apparu que l'angle formé à l'intersection des lignes équipotentielles et de la surface libre constituait le critérium décisif de la justesse d'une solution.

\section{B) Calcul de cas d'essais caractéristiques.}

1. Présentation. - Les figures 17 à 19 représentent la solution par voie de relaxation, respectivement dans le cas des essais 2,4 et 6 de la série A.

Le réseau constitue l'image d'une section radiale du modèle du puits dont l'axe est à gauche. Sur cet axe sont portées des hauteurs mesurées audessus de la base du modèle; en dessous du réseau on trouve l'échelle des distances radiales, en pouces, à partir de l'axe du puits. Sur chaque figure sont portés les niveaux de l'eau à l'entrée et à la sortie. La surface libre et les neuf lignes équipotentielles qui partagent l'écoulemenl en dix intervalles équivalents, sont tracées en gros trait continu. La ligne supérieure de la frange capillaire est représentée par un gros trait interrompu. Les brins du réseau de relaxation figurent en traits plus fins : continus pour les brins entiers, interrompus pour les demi-brins. Les hauteurs piézométriques correspondant aux différentes lignes équipotentielles sont marquées en pouces d'eau, gros caractères, à côté de chaque ligne; les valeurs nodales finales de la hauteur piézométrique sont portées en petits caractères au-dessus et à gauche des nœuds du réseau. Pour toutes les valeurs nodales, l'unité de mesure adoptée est le dixième de pouce d'eau, ce qui est la limite de précision qu'on peut atteindre dans les lectures piézométriques. Pour faciliter les calculs, nous avons pris comme longueur du côté de la plus grande maille carrée du réseau le rayon même du puits; dans le cadre de la méthode de relaxation, nous avons adopté, comme unité de distance radiale, la demi-longueur du côté du plus petit carré du réseau figurant dans la solution $\left({ }^{*}\right)$.

2. Conditions aux limites. - La figure 20 est un croquis schématique d'une section radiale semblable à celles qui sont représentées sur les figures 17 à 19.

La ligne CDE représente la section d'entrée, la ligne BAHGF la section de sortie. La ligne BC correspond à la base du modèle. Le plan d'eau à l'entrée est indiqué en $\mathrm{D}$ à une hauteur $\mathrm{H}$ au-dessus de la base; à la sortie, le plan se trouve en $A$, à une hauteur $h_{0}$ au-dessus de la base. La ligne DNG représente la surface libre; la ligne EPF est la limite supérieure de la surface de suintement. Les axes coordonnés, $r$ (distance radiale) et $z$ (hauteur), correspondent respectivement à la base et à l'axe du puits; la cote du point d'intersection de la surface libre et de la section de sortie est désignée par $z_{0}$

$\left.{ }^{\star}{ }^{\star}\right)$ Les distances radiales n'interviennent que dans l'élimination des valeurs résiduelles. 
Les conditions aux limites dans le cas présent, et la forme sous laquelle elles ont servi à l'étude des essais 2,4 et 6 de la série $A$, peuvent etre exprimées de la façon suivante :

(1) De A à B :

$0=h_{0}$, où 0 est la hauteur piézométrique; ceci exprime que la courbe $\mathrm{AB}$ est la dernière ligne équipotentielle dans le sens de l'écoulement.

(2) De B à C :

$d \varphi / d z=0$; ceci exprime que la courbe BG est une ligne de courant et que toutes les lignes équipotentielles doivent la couper à angle droit.

(3) De C à D :

$0=\mathrm{H}$, autrement dil la courbe $\mathrm{CD}$ est la première ligne équipotentielle dans le sens de l'écoulement.

(4) De D en G (par E, P et F) :

$\varphi=z-h_{c}$ et $d \varphi / d n=0$, où $h_{c}$ désigne la remontée capillaire et $n$ la direction normale à la courbe limite en chaque point.

La première de ces conditions résulte de ce que la surface libre DNG doit satisfaile à la relation $0=z$; il s'ensuit que la hauteur piézométrique en un point de la limite supérieure de la frange capillaire devra être inférieure à la cote de ce point, de la valeur correspondant à la tension capillaire te l'eau. La seconde condition résulte de ce que la courbe DEPFG est une ligne de courant.

(5) De G à A :

Le point $G$ étant sur la surface libre, la hauteur piézométrique est égale à la cote $z_{0}$ et la pression de l'eau est nulle en ce point. 'Toutefois, sur unc certaine hauteur en dessous du point $\mathrm{G}$, aucun écoulement ne se manifestera à l'extérieur du sable car la tension superficielle du fluide crée une contrepression s'opposant à la tendance du fluide à suinter à travers la paroi. Quant la pression de l'eau a suffisamment augmenté pour surmonter cette contrepression (par exemple au point $H$ ), de l'eau suintera de la masse de sable à ce niveau.

La figure donne un schéma de la distribution des pressions de l'eau le long de la surface verticale FGHAB. Le point $F$, où la ligne de courant marque un angle droit, constitue un " angle mort ». En cet endroit, la dépression capillaire est maximum, puis elle diminue vers le bas, devient une pression en un certain point $G$ et finalement atteint une valeur (par exemple $\bar{h}_{m a x}$ ), suffisante pour surmonter la résistance à l'écoulement latéral que crée la tension superficielle au niveau de la surface du sable. La variation de la pression du fluide le long de FGH est moins rapide que celle qui correspond à la distribution hydrostatique, car une partie du potentiel total intervient pour maintenir l'écoulement le long de la ligne de courant FGH. La distance FGH est donc moindre que la somme $\left(h_{r}+\bar{h}_{\text {max }}\right)$ et $\bar{h}_{\text {max }}$ est bien moindre que $h_{c}$.

La partie restante de la limite (ligne HA) constitue la surface de sortie. Comme cependant la pression du fluide en $\mathrm{H}$ est supérieure à zéro, la simple relation $0=z$, ne s'applique pas à la totalité de la distance HA. Il semble logique d'admettre que l'excédent de pression se dissipe sur une petite distance en dessous du point $H$, et que la majeure partie de la ligne $H A$ satisfait à la relation en question.
On peut donc résumer de la façon suivante les conditions aux limites entre $\mathrm{G}$ et $\mathrm{A}$ :

Entre G et $\mathrm{HI}: d \varphi / d r=0$, car la majeure partie de la ligne est une ligne de courant.

$$
\text { De } \mathrm{H} \text { en } \mathrm{A}: \varphi=z+h^{\prime} \text { avec } \bar{h}_{\text {max }} \geqslant h^{\prime} \geqslant 0 \text {, }
$$

ce qui veut dire que HA n'est ni une ligne de courant ni une ligne équipotentielle. On ne saurait non plus la définir comme une surface libre, sauf dans la portion qui ne subit pas l'excédent de pression dont le maxinum est atteint au point $\mathrm{H}$.

3. MÉthode aDOptée. - Nous avons utilisé pour les trois solutions considérées les valeurs numériques suivantes : $h_{c}=3,6$ pouces $(9,2 \mathrm{~cm})$;

$$
\bar{h}_{m a x}=1,0 \text { pouce }(2,54 \mathrm{~cm})
$$

La hauteur $h_{c}$ de la remontée capillaire a été déterminée en mesurant, sur les figures 12 à 14, la projection sur une verticale de la portion d'une ligne équipotentielle, comprise entre la limite supérieure de la frange capillaire et la surface libre.

La valeur de $\vec{h}_{m a}$ a été choisie d'après certaines expériences auxiliaires réalisées sur des modèles bi-dimensionnels à petite échelle, constitués de sable disposé entre deux panneaux en bois écartés d'un pouce $(2,54 \mathrm{~cm})$.

Au point' de départ de la méthode de relaxation il fallait placer de facon arbitraire le point $\mathrm{H}$; on s'est d'ailleurs appuye sur cette méthode pour juger du choix du point $H$ ainsi que de la valeur de $\bar{h}_{m \ldots r}$; on ne pouvait en effet obtenir la précision voulue, dans l'une et dans l'autre de ces déterminations, que par des tâtonnements.

4. Discussion. - Dans la discussion qui va suivre, la figure 20 sera identifiée aux figures 17 à 19 ; les points, les distances et les charges marqués sur la figure 20 seront donc utilisés pour désigner sur les figures 17 à 19 les éléments correspondants, sans être identifiés autrement.

Ce qu'il $\mathrm{y}$ a de plus saillant dans les solutions obtenues par la méthode de relaxation, dans le cas des essais 2, 4 et 6 de la série $A$, est la disposition du réseau d'écoulement à la partie supérieure de la section aval. Bien que la ligne DEPFGH soit une ligne de courant, on notera que sa pente devienl nulle aux abords du point $F$ et qu'en ce point elle tourne de $90^{\circ}$. Le point $F$ est done un « angle mort $\gg$.

Sur les figures 17 a 19 la contre-pression semble déformer la surface libre á peu près comme elle le fait pour la ligne de courant limite. A noter cependant qu'avec les conditions aux limites adoptées ici, la surface libre n'est pas une ligne de courant, comme elle ne l'est pas non plus sur le modèle.

La figure 21 représente deux réseaux d'écoulement déterminés par la méthode de relaxation pour un cas correspondant à l'essai 4 de la série $A$. Les courbes en traits pleins correspondent au réseau de la figure 18, les courbes en tirets montrent l'allure du réseau quand on néglige les effets de la frange capillaire ef de la contrepression. On peut 
voir que la distorsion due à la frange capillaire et à la contrepression ne se fait sentir que sur une zone rulativement petite de la partie supérieure du réseau près de la section de sortie.

Dans ce groupe de solutions, $h_{r}$ et $\bar{h}_{\max }$ avaient des valeurs relativement faibles, en raison de la grosse granulométrie du sable. Ces valeurs seraient plus grandes avec des matériaux plus fins, qui sont aussi les plus fréquents.

Pour situer le point $H$ (fig. 20 ) dans une solution particulière, on devra procéder par tâtonnements. Après avoir choisi le point et lui avoir assigné la valeur appropriée de la hauteur piézométrique $\left(\varphi=z+\bar{h}_{\text {max }}\right)$, on pourrait trouver la solution par la méthode de relaxation, et la vérifier ensuite par observation directe des valeurs relatives de $\mathrm{GH}$ et HA, mais la disposition du modèle s'y opposait. On devait donc, dans l'étude des essais 2,4 et 6 de la série A, commencer par situer $H$ d'après les résultats de certains essais complémentaires et régler ensuite sa position d'après le déroulement de la méthode de relaxation.

\section{C) Comparaison des résultats expérimentaux avec les solutions fournies par la méthode de relaxation.}

1. Présentation de la Question. - Sur les figures 22-24, les solutions obtenues par la méthode de relaxation dans les cas 2,4 et 6 de la série $A$ sont superposées aux tracés des essais. La limite supérieure de la frange capillaire n'est pas comprise dans la comparaison, car la correspondance entre l'expérience et la théorie dans le cas de cette ligne suit toujours de très près la correspondance obtenue pour la surface libre.

L'écart entre les différentes lignes équipotentielles théoriques et les courbes expérimentales correspondantes est exprimé en \% du potenticl théorique, et noté aux extrémités supérieure et inférieure des courbes expérimentales. Presque toujours ces valeurs représentent l'écart maximum entre l'expérience et la théorie pour la ligne équipotentielle considérée.

2. Surfaces libres. - Dans les trois cas considerés on observe une concordance très serrée entre surface libre théorique et expérimentale. Dans chaque cas, l'écart le plus grand se produit au point d'intersection de la surface libre et de la paroi du puits. En cet endroit, la courbe théorique s'incurve assez brusquement et revient à une pente sensiblement horizontale, tandis que la courbe expérimentale n'a pas ce coude final. On pourrait expliquer cette différence en partie par le fait que, précisément sur les deux derniers pouces $(5 \mathrm{~cm})$, où se trouve le coude, les lectures piézométriques ont été rares. Aux tracés expérimentaux de la surface libre on pourrait adjoindre, en bout, un petit fragment de courbe inverse ne contredisant pas les lectures jiézométriques; ce procédé ne saurait cependant être justifié par ces seules considérations expérimentales.

3. Lignes ÉQuipotentielles. - Les figures 22-24 montrent que les écarts maximums entre équipotentielles observées et théoriques correspondantes, se produisent dans les trois ou quatre dernières bandes du réseau précédant la paroi du puits; l'écart le plus grand ayant lieu entre les deuxième et quatrième bandes.

Le dégagement d'air constituant la principale cause des écarts entre réseaux observés et réseaux théoriques, on pourrait s'attendre à ce que les écarts soient surtout grands là où la pression du fluide est la plus basse, ou là où le gradient est le plus fort. Or, les pressions les plus basses se présentaient le long de la surface libre et de la paroi du puits, alors que les gradients les plus élevés se trouvaient à l'intersection de la surface de suintement et du plan d'eau dans le puits. Il est donc évident que les régions de pression minimum et de gradient maximum ne coïncident pas entièrement avec les régions où sont relevés les plus grands écarts entre réseau expérimental et réseau théorique; et on en déduit que c'est la façon d'évaluer les écarts en \% qui peut, dans une certaine mesure, être une cause d'erreurs. Par exemple, si au lieu de rapporter à zéro les écarts entre hauteurs piézométriques expérimentales et théoriques, on les rapportait à $h_{0}$, valeur minimum dans chaque cas, le pourcentage d'écart maximum se trouverait déplacé vers la section aval. Et si les écarts en \% étaient calculés par comparaison des gradients observés aux gradients théoriques, la déviation dans le même sens serait bien plus marquée. Néanmoins, les écarts déduits de la comparaison des gradients ne pourraient être pris en considération qu'avec beaucoup de prudence du fait que les équipotentielles expérimentales sont, dans tous les cas, des lignes continues, tracées en suivant, d'aussi près que possible, une série de points résultant de l'interpolation des lectures piézométriques.

4. Hauteur piézométrique sur le fond. - La figure 25 met en regard les courbes de la hauteur piézométrique sur le fond obtenues par la méthode de relaxation et celles qui se déduisent des mesures. Sur chaque paire de courbes est marqué l'écart en \% entre courbe théorique et courbe expérimentale. Ces valeurs ont été relevées aux points où l'écart est maximum.

On remarque sur cette figure que la relation entre les hauteurs piézométriques observées et théoriques caractérise l'allure générale de l'ensemble du réseau. Les écarts entre valeurs observées et théoriques vont en augmentant d'un essai à l'autre; leur maximum est voisin de $5 \%$.

En comparant la figure 17 à la figure 25 on s'aperçoit que les courbes des hauteurs piézométriques obtenues par la méthode de relaxation concordent mieux avec les observations que la courbe de DupuIT-THIEM. 


\section{V. - RÉSUMÉ ET CONCLUSION $(*)$}

\section{A) La méthode de relaxation}

Parmi les essais sur modèle qui ont fait partie de la présente recherche, trois cas caractéristiques ont été choisis et soumis à la méthode de relaxation.

Nos analyses diffèrent des analyses antérieures consacrées à des problèmes semblables en ce sens que l'effet de la tension superficielle du fluide sur le réseau d'écoulement fut pris en compte d'une manière que nous estimons qualitativement exacte si, quantitativement, elle est seulement approchée. Il est à supposer qu'à cette tension superficielle sont dues deux particularités importantes du réseau : la frange capillaire d'une part, et, d'autre part, par suite de la contre-pression s'opposant à l'écoulement vers l'extérieur, l'absence de débit constatée à la section de sortie, sur une certaine hauteur en dessous de la surface libre. Ces deux effets ont pu être évalués d'après les observations faites pendant les essais sur le modèle et d'après quelques essais complémentaires.

La concordance constatée entre les résultats des essais et ceux de la méthode de relaxation est assez bonne. En se basant sur ce groupe de comparaisons, on peut affirmer que l'écart maximum à prévoir entre les hauteurs piézométriques réelles et les hauteurs calculées serait d'environ $6 \%$, dans tous les problèmes concernant l'alimentation d'un puits gravitaire.

La méthode de relaxation peut donc être considérée comme un procédé analytique sûr, chaque fois où l'on a à déterminer le réseau entourant un puits gravitaire. De plus, cette étude de l'alimentation d'un puits a mis en évidence la souplesse de cette méthode d'analyse dans le maniement des conditions aux limites qui rendent si difficiles l'application des méthodes classiques.

\section{B) Surface libre}

1. GÉNÉRALITÉs. - La conception théorique traditionnelle de la surface libre est celle d'une ligne de courant délimitant de façon bien marquée la partie mouillée et la partie non mouillée d'un milieu poreux où se produit un écoulement gravitaire.

L'analyse par la méthode de relaxation et, d'autre part, les observations effectuées pendant notre campagne d'essais lui enlèvent une partic de son caractère. Ce n'est ni une ligne de démarcation, ni

$\left(^{*}\right)$ Dans ce résumé de nos travaux, nous avons complètement écarté toute comparaison de nos résultats avec ceux obtenus par BabBitr et Caldwel, par Muskat, par YANG et par d'autres dont les publications sont antérieures à 1951. Cependant, puisque les références à ces divers chercheurs sont données, il est peut-être bon, afin d'être complet, d'ajouter que de nombreuses recherches publiées plus récemment se rapportent au même problème. Deux en particulier se rapprochent beaucoup de la présente étude à la fois par leur objet et leur importance : ce sont celles de Boultor [8] et dic Hansen [9]. une ligne de courant; c'est simplement une ligne particulière d'égale pression. Elle n'en est pas moins une ligne d'égale pression importante, parce que c'est en s'y rapportant qu'on arrive à mieux définir la limite supérieure de la frange capillaire et aussi parce que cette conception de la surface libre, limite supérieure de l'écoulement, peut être adoptée dans la pratique courante.

L'aspect de la surface libre vers la paroi du puits nous est apparu quelque peu différent, dans le détail, de ce qu'on se représentait habituellement. Ce n'est pas une ligne de courant, et à cause de son « angle mort », elle n'aborde pas la paroi du puits en incurvant sa tangente comme dans le cas où le fluide filtrant n'aurait pas de tension superficielle. Elle ne coupe pas non plus à angle droit les lignes équipotentielles.

L'étude de la question a également montré que la tension superficielle détermine un relèvement de la surface libre sur tout son parcours d'une quantité dont le maximum a lieu à la paroi du puits.

2. Equations empiriques. - Du moment qu'il est possible d'obtenir une bonne concordance entre résultats théoriques et expérimentaux, il serait utile de préciser les équations permettant de situer et de dessiner la surface libre à partir des rapports caractéristiques appropriés. Nous nous proposons donc d'établir des équations empiriques d'après les résultats de nos recherches.

La première équation exprime la hauteur de la surface de suintement. Rapportée, comme d'ordinaire, à une surface cylindrique, elle s'écrira :

$$
\left(\frac{z_{0}-h_{0}}{\mathrm{H}-h_{0}}\right)=\frac{\left[1-\left(h_{0} / \mathrm{H}\right)^{2,4}\right]}{\left[1+(1 / 50) \log _{e}\left(\mathrm{R} / r_{0}\right)\right]\left[1+5 /\left(\mathrm{H} / r_{0}\right)\right]}
$$

les symboles indiquent les distances marquées sur la figure 20 .

D'après la forme du second membre de l'équation (4) on voit que le plus important des trois rapports caractéristiques est $h_{0} / \mathrm{H}$; vient ensuite $\mathrm{H} / r_{0}$ et finalement $\mathrm{R} / \mathrm{r}_{\mathrm{n}}$.

La figure 26 est un abaque servant à la résolution de l'équation $(4)$. $\left(\mathrm{R} / r_{0}\right)$ étant le rapport dont les variations se répercutent le moins sur $\left(z_{0}-h_{0}\right) /\left(\mathrm{H}-h_{0}\right)$, sa valeur a été fixée à 500 pour toutes les courbes. On pourra se servir de cette figure pour déterminer la valeur que prend le rapport $\left(z_{0}-h_{0}\right) /\left(\mathrm{H}-h_{0}\right)$ pour toutes les combinaison; possibles des rapports caractéristiques, si l'un d'entre eux est $\mathrm{R} / \mathrm{r}_{0}=500$. Pour des valeurs de $\mathrm{R} / \mathrm{r}_{0}$ autres que 500 , un coefficient de correction (d'ordinaire insignifiant), peut se deduire facilement de la forme de l'équation (4).

Mais cette équation ne définit que l'extrémité de la surface libre; aussi, pour exprimer l'ensemble de son tracé, on utilisera l'équation :

$\frac{z-z_{0}}{\mathrm{H}-z_{0}}=2,5\left(\frac{r-r_{0}}{\mathrm{R}-r_{0}}\right)-1,5\left(\frac{r-r_{0}}{\mathrm{R}-r_{0}}\right)^{1 . \overline{3}}$ 
La figure 27 établit la comparaison de la courbe exprimée par l'équation (5) avec les surfaces libres théoriques correspondant aux essais 2,4 et 6 de la série A. Les tracés de la surface libre construits d'après les équations de BaBbitt et Caldwell [1] y figurent également.

Lors de l'application des équations (4) et (5) à des cas particuliers, il est à conseiller de se servir d'observations ou de la courbe de Dupurt-Thiem (à une distance du puits, de par exemple $50 r_{0}$, où les hypothèses de Dupurt sont valables) pour déterminer les coordonnées $(r, z)$ d'un point, et les substituer, dans les équations empiriques, aux points (R. H.). La surface libre correspondant aux équations (4) et (5) formera alors le troncon final, à raccorder à l'extrémité aval d'une surface libre dont la partie restante serait déduite de l'équation DupurTTHrem avec une précision convenable.

\section{C) La frange capillaire}

Nous avons examiné, au chapitre précédent, l'influence qu'exerce sur la position de la surface libre la frange capillaire.

D'autre part, la contribution apportée au débit par cette frange a été exprimée par l'équation (1). Le facteur 2 qui y figure peut être comparé à la valeur 1 suggérée par Muskat [3]. Ùne étude plus approfondie fournirait peut-être une valeur intermédiaire.

\section{D) Hauteur piézométrique sur le fond.}

Les travaux de YANG [7] et les résultats déduits de la présente étude montrent que l'équation de Dupuit-THIEM constitue une approximation satisfaisante des variations de la hauteur piézométrique sur le fond; toutefois, la concordance n'est peut-être pas aussi bonne que tendaient à le montrer certains travaux antérieurs.

\section{E) Degré et uniformité de saturation}

On est amené à admettre que le degré de saturation n'affecte pas considérablement l'allure du réseau; tandis que la plus ou moins grande uniformité dans la distribution des poches d'air joue un grand rôle.

\section{F) Débit}

D'autres investigateurs ont déjà démontré la validité de l'équation de Dupuí-Thiem pour le calcul du débit et du coefficient de perméabilité moyen. Les résultats obtenus au cours des travaux présentés ici ajoutent encore au poids de cette constatation.

\section{REMERCIEMENTS}

Le mémoire qu'on vient de lire a été rédigé d'après une thèse présentée par l'auteur à la Faculté des Arts et Sciences de l'Université de Harvard, à titre de contribution partielle pour l'obtention du grade de docteur ès sciences (Génie Civil).

L'auteur exprime sa reconnaissance au Prof. Arthur CaSagrande qui lui a indiqué le problème à résoudre et qui l'a guidé dans ses recherches; au Prof. S. D. WILson pour son aide et ses conseils aussi bien dans la phase théorique que dans la phase expérimentale de ses travaux; au Prof. Harold A. Thomas pour ses nombreux et si utiles conseils; à M. M. P. Grotjohan et R. Olsson pour l'aide fournie pendant la construction du modèle à la Soil Mechanichs Research Fund de l'Université de Harvard enfin pour lui avoir fourni les fonds nécessaires. Les dessins accompagnant ce texte ont été exécutés par Mme Margaret M. Nergr.

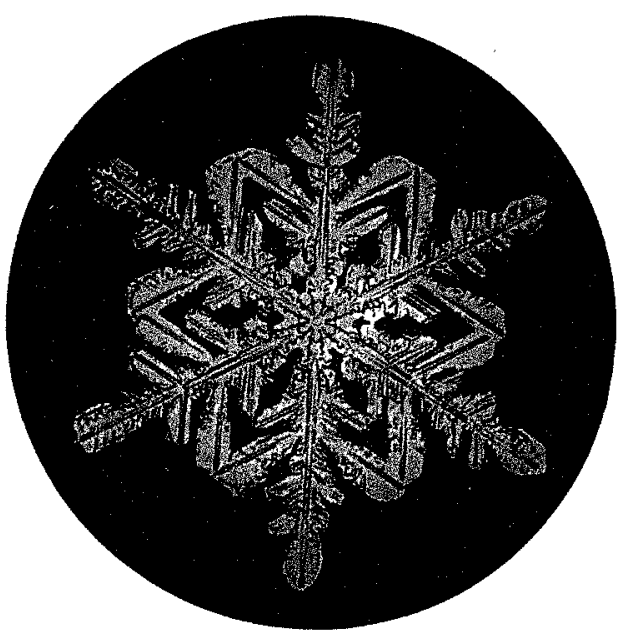

